\title{
A NEW AUV PLATFORM FOR STUDYING NEAR SHORE BIOLUMINESCENCE STRUCTURE
}

\section{S BLACKWELL ${ }^{1}, \mathrm{~J} \mathrm{CASE}^{2}, \mathrm{~S} \mathrm{GLENN}^{3}, \mathrm{~J} \mathrm{KOHUT}^{3}, \mathrm{M} \mathrm{MOLINE}^{1}$, M PURCELL ${ }^{4}$, O SCHOFIELD $^{3}$, C VONALT $^{4}$}

${ }^{1}$ Biological Sciences, Cal Poly State University, San Luis Obispo, CA 93407, USA

${ }^{2}$ MSI, University of California Santa Barbara, Santa Barbara, CA 93106, USA

${ }^{3}$ IMCS, Rutgers University, New Brunswick, NJ 08901, USA

${ }^{4}$ Woods Hole Oceanographic Institution, Woods Hole, MA 02543, USA

\section{INTRODUCTION}

Peaks in bioluminescence are often associated with small spatial features including frontal boundaries, vertical thin-layers, and convergence/divergence zones. ${ }^{1,2}$ Due to the highly variable nature of these features in the near shore environment, accurate assessment of bioluminescence potential is dependent upon the application of appropriate platforms and the use of these platforms at relevant time and space scales. Both traditional shipboard profiling techniques and moored vertical profiling instrumentation fail to adequately provide the appropriate spatial resolution to explain the dynamics of near shore bioluminescence. In an effort to resolve the mechanisms underlying the temporal and spatial variation in near shore bioluminescence, this study integrates an autonomous underwater vehicle (AUV) into a multi-platform adaptive sampling network. More specifically, we utilize the AUV to examine the effect of tides on the location and structure of optical, biological and physical signatures of a frontal zone in time and space. Furthermore, we examine the importance and significance of optimising the AUV flight paths to quantify small-scale structure in near shore environments.

\section{MATERIALS AND METHODS}

On July 21, 2001 at the LEO-15 site off the coast of New Jersey (Fig. 1a) a frontal feature was identified at 21:00 by the R/V Caleta using an FSI MiniBat towed undulating instrument system. Coastal Ocean Doppler Acoustic Radar (CODAR) was used to monitor surface currents and to identify the time of maximal offshore tidal flow. Higher resolution sampling of the frontal zone was conducted later that evening using a modified REMUS (Remote Environmental Measuring UnitS) AUV. In addition to CTD sensor and Acoustic Doppler Current Profiler (ADCP), the newly developed AUV incorporates a fluorometer, optical backscatter sensor and bathyphotometer into a modified nosecone. The bathyphotometer ${ }^{3}$ pulls water through a $0.5 \mathrm{~L}$ detection chamber with a residence time of $1 \mathrm{sec}$. A photomultiplier tube incorporated into the chamber measures the bioluminescence potential of the passing water. The AUV also measured temperature (T), fluorescence (FL) optical backscatter (OBS), current direction and current magnitude. The vehicle was 

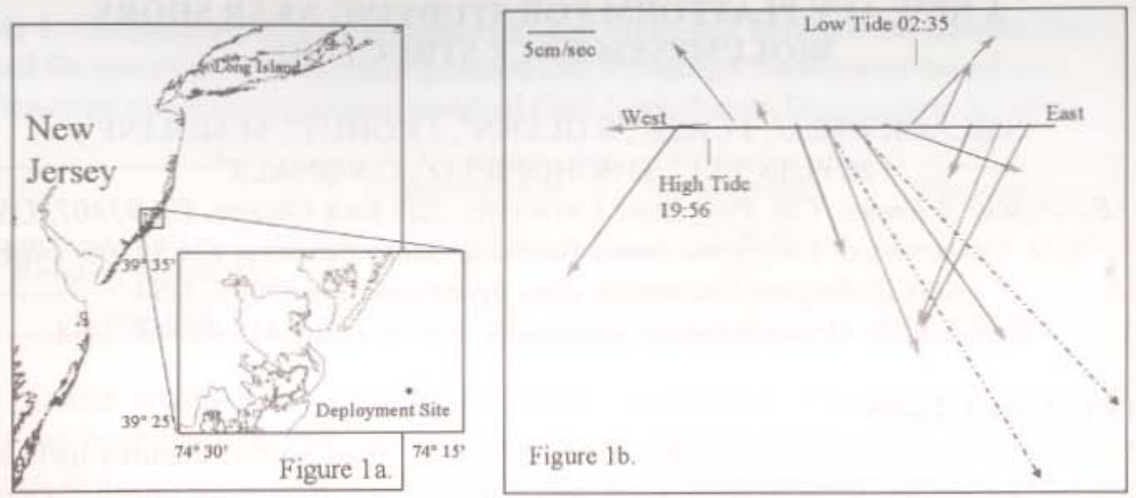

Figure 1. a.) Map of New Jersey and study location. b.) Vector plot of surface current magnitude and direction at site over time. Hashed arrows show hourly averages from the vehicle's ADCP, solid lines are hourly CODAR averages.

deployed at 22:42 and made four passes along a $3 \mathrm{~km}$ transect, undulating between 3 and 9 meters, completing its mission at $00: 31$ on July 22, 2001.

\section{RESULTS AND CONCLUSIONS}

Surface currents as measured by CODAR were correlated with those measured by the AUV ADCP, and show mission deployment time corresponded with maximal offshore tidal flow between 22:00 and 00:00 (Fig. 1b). Figure 2 illustrates the effect of the extreme offshore pull of the tidal currents, where the spatial features of T, BL, FL, and OBS can be seen migrating overtime in an offshore direction. This is particularly evident in T and BL between 6 and $9 \mathrm{~m}$, and in the uppermost offshore corner of both FL and OBS plots. It is of interest to note the lack of coherence between the FL and BL signals (Fig. 2b \& 2c). This lack of coherence strongly suggests the bioluminescent community was dominated by heterotrophs. The fact that BL increased at increasingly shallower depths over time (Fig. 2b) further suggests the bioluminescent community is likely dominated by vertically migrating, zooplankton or heterotrophic dinoflagellates.

Further analysis of the data revealed a dissipation of the front in conjunction with the ebbing tide (Fig. 3a). The observed decrease in normalized standard deviation for T, BL and FL over time indicates that the differences in physical and biological signatures, which are usually associated with a frontal zone, were decreasing in intensity as the tide is receding. OBS did not exhibit this same decrease in normalized standard deviation over time. However, it is likely that this increase in standard deviation was due to the highly turbid mass of water moving into the study region from onshore as the tide is receding (Fig. 2d). 

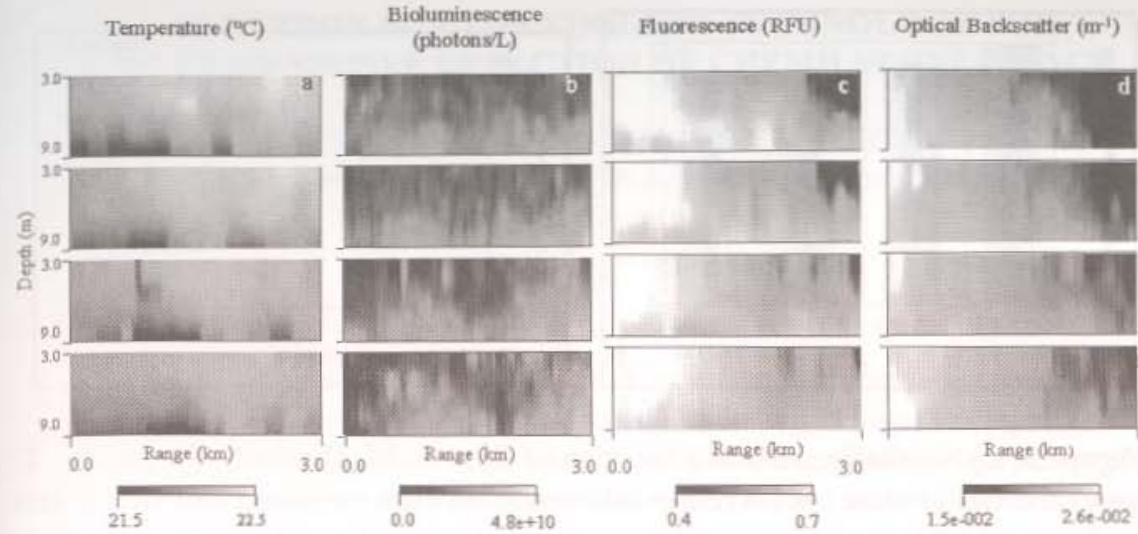

Figure 2. Spatial distribution of T, BL, FL and OBS during four consecutive passes of the transect. Panels progress temporally as you move down.

Although results illustrated the effect of the tide on a frontal zone, the question remains as to whether the dynamics were adequately resolved? In order to answer this question flight path optimisation was quantified by running virtual missions of variable ascent/descent rates through a highly structured fluorescence data matrix (http://marine.rutgers.edu/mrs/hycode2/data/jul23/01072303.flu.gif, range scaled to $1 \mathrm{~km})$. Virtual flights were made through the data matrix at six different ascent/descent rates ranging from $3 \mathrm{~m} / \mathrm{min}$ to $22 \mathrm{~m} / \mathrm{min}$. Virtual AUV data was then interpolated and compared to the measured fluorescence matrix. Simulations of a shipboard profiling system were also conducted with differences calculated between interpolated profile data and the fluorescence matrix.

Average differences for each of the six flight paths as calculated from the flight path optimisation exercises fit a logarithmic curve $\left(\mathrm{R}^{2}=0.98\right)$ and suggest that the ability of the vehicle to resolve small-scale structure is most efficient at an ascent/descent rate of $\sim 10 \mathrm{~m} / \mathrm{min}$ (Fig. 3b). Because these exercises were carried out in a highly structured water mass, $10 \mathrm{~m} / \mathrm{min}$ represents a conservative estimate. In less complex waters it is probable that slower ascent/descent rates would be sufficient. Data for this study was collected at a rate of $6 \mathrm{~m} / \mathrm{min}$, suggesting some of the fine scale structure may have been missed. The fact that the average difference between shipboard profiled data and the actual data field was 80 RFU (Relative Fluorescence Units), at least double the average difference obtained through five of the six AUV simulations, emphasizes the inadequacy of traditional shipboard profiling measurements in characterizing small-scale near shore dynamics. 

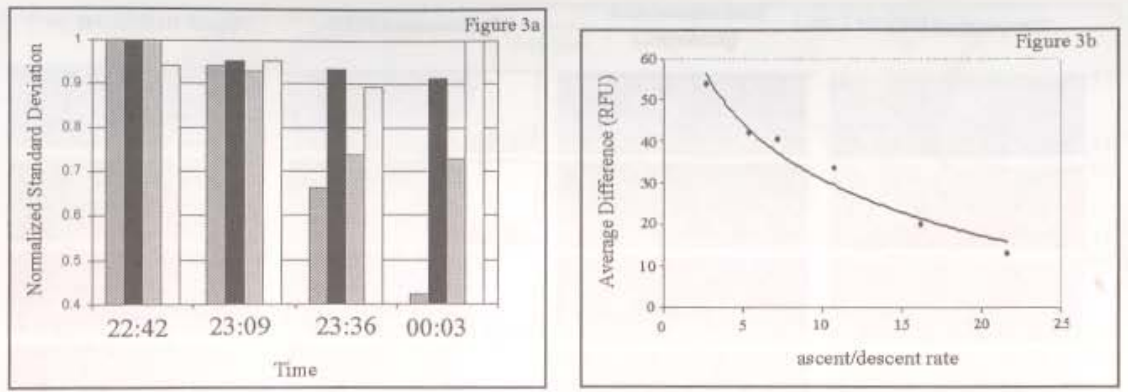

Figure 3. a.) Normalized SD as a function of time for BL (hashed), FL (black), T (grey) and OBS (white). b.) Average difference between measured and virtual data matrices as a function of ascent/descent rates, shipboard profile data not shown.

In conclusion this study demonstrated the utility of adaptive sampling networks and highlighted their importance in characterizing small-scale bioluminescence structure in near shore environments. Data collected by the R/V Caleta, CODAR and vehicle $A D C P$ were critical to our ability to characterize the effect of tides on the physical, biological and optical signatures in a frontal zone. As is evidenced in this study, circulation patterns, even on very small temporal and spatial scales can play a significant role in the physical, optical and biological structure in the near shore environment. Moreover this study demonstrates the successful integration of an AUV into a sampling network as an appropriate platform for studying small-scale bioluminescence structure.

\section{ACKNOWLEDGEMENTS}

Research funded by NASA grant NAG5-8674 and ONR grant N000-14-00-0570. Special thanks to Cyril Johnson, Matt Oliver and Cristina Orrico.

\section{REFERENCES}

1. Losee J, Lapota D, Lieberman S. Bioluminescence: A new tool for oceanography. In: Zirano A, editor. Mapping Strategies in Chemical Oceanography. Washington: American Chemical Society, 1985: 211-33.

2. Widder E, Johnsen S, Bernstein S, Case J, Neilson J. Thin layers of bioluminescent copepods found at density discontinuities in the water column. Mar Biol 1999; 134:429-37.

3. Moline M, Heine E, Case J, Herren C, Schofield O. Spatial and temporal variability of bioluminescence potential in coastal regions. In: Case JF, Herring PJ, Robison BH, Haddock SHD, Kricka LJ, Stanley PE, editors. Proceedings of the $11^{\text {th }}$ International Symposium on Bioluminescence \& Chemiluminescence. Singapore: World Scientific, 2000: 123-6. 